\title{
Analytical Model of IEEE 802.15.4 Non-beacon Mode with Download Traffic by the Piggyback Method
}

\author{
Tae Ok Kim ${ }^{1}$, Jin Soo Park ${ }^{2}$, Kyung Jae Kim ${ }^{1}$, and Bong Dae Choi ${ }^{1}$ \\ ${ }^{1}$ Department of Mathematics and Telecommunication Mathematics Research Center, \\ Korea University, Seoul, Korea \\ \{violetgl, kimkjae, queue\}@korea.ac.kr \\ ${ }^{2}$ USN Service Division, KT, Seoul, Korea \\ vtjinsoo@paran.com
}

\begin{abstract}
We analyze the MAC performance of the IEEE 802.15.4 LRWPAN non-beacon mode with the piggyback method in non-saturated condition. Our approach is to model a stochastic behavior of one device as a discrete time Markov chain. We propose an analytical model describing the download behavior of a device using piggyback method. We obtain the performance measures such as throughput, packet delay, energy consumption and packet loss probability of a device. Numerical results and simulation results show that the piggyback method which removes a backoff procedure in the backoff method can reduce the delay, loss probability and energy consumption compared with backoff method. Our results can be used to find the optimal number of devices with some constraints on packet delay and packet loss probability.
\end{abstract}

Keywords: IEEE 802.15.4, Piggyback Method, Performance Analysis.

\section{Introduction}

IEEE 802.15.4[1],2] is a standard toward low complexity, low power consumption and low data rate wireless data connectivity. Therefore IEEE 802.15.4 will play a key role as transmission protocol at WSN(Wireless Sensor Network) where energy consumption is an important factor.

Diverse applications for wireless sensor network based on IEEE 802.15.4 have generated interest in analytical models of access mechanism based on CSMA/CA. Pollin et al. [5] and Park et al. 6] proposed analytical model for upload traffic on IEEE 802.15.4 beacon-enabled mode under saturated condition where devices have always packets to send. In real environment, packets are generated in not too often, so that a device will have no packets to send or receive for significant periods. Therefore we need to investigate non-saturated case where a device does not have packets to send or receive for some period of time. Misic et al. [7] analyzed performance of IEEE 802.15.4 with both upload and download traffic in beacon-enabled mode under non-saturated condition. 
In non-beacon mode, for download data transfers, a device periodically sends a download request packet using CSMA/CA to its coordinator and the coordinator sends an acknowledgment packet to indicate the successful reception of the download data request. The standard of IEEE 802.15.4 specifies two ways to send download data packet. One way is to send download data packet by following backoff procedure using CSMA/CA (See Fig. 1(a)). Such a method is called the backoff method and is called the method with CSMA/CA in the standards. Other way is to send download data packet immediately after ACK packet is sent (i.e. without backoff procedure using CSMA/CA). Such a method is called the piggyback method in this paper, and is called the method without CSMA/CA in the standards. The piggyback method is obtained by removing the backoff period from the backoff method, the piggyback method can shorten the delay of download data packet and so can save the energy consumption. Another merit of piggyback method is that there is no loss of download data packet. We assume that all devices are synchronized, so that the slotted CSMA/CA is applied. Therefore, in order to protect acknowledge packets, implementation of two consecutive CCA (clear channel assessment) is assumed. Only these assumptions which are slotted CSMA/CA and two consecutive CCAs are different from the standard of IEEE 802.15.4 where unslotted CSMA/CA and only one CCA is used in non-beacon mode. Kim et. al. 4] analyzed the performances of IEEE 802.15.4 with backoff method under these assumptions.

In this paper, we investigate the performance of IEEE 802.15.4 with piggyback method and compare performance measures of the piggyback method and backoff method [3]. We model the stochastic behavior of a device with download traffic as a discrete-time Markov chain. First, we propose an analytical model describing the download behavior of a device using piggyback method. Then, we obtain the performance measures such as throughput, packet delay, energy consumption and packet loss probability of a device with download traffic. $\mathrm{Nu}-$ merical results and simulation results show that by removing a backoff procedure in the backoff method, the piggyback method can reduce the delay, loss probability and energy consumption compared with backoff method. Our results can be used for determining the optimal number of devices which can be admitted to the system while supporting the required QoS on the expected packet delay and the packet loss probability.

This paper is organized as follows. We describe the MAC procedure for download in Section 2, In the Section 3.1, we propose the analytical model of a device with download traffic only under non-saturated condition and obtain performance measures from our analysis. Numerical and simulation results for performance measures of the network with download traffic are presented in Section 4 .

\section{MAC Procedure for Download by Piggybacked Method}

When the coordinator wishes to transfer data to a device, it stores the download data packet and waits until the appropriate device makes a contact and requests the data. A device may make contact by transmitting a download request packet 


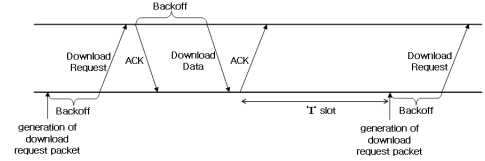

(a) Backoff method

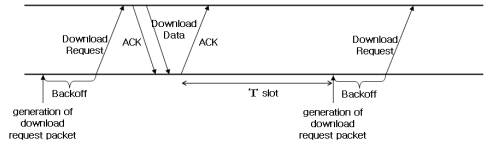

(b) Piggyback Method

Fig. 1. Download procedure in a non-beacon network

using CSMA/CA periodically. The coordinator sends an acknowledgment packet which notifies the successful reception of the download request packet and information on whether there is a download data packet. If data are pending, the coordinator transmits the download data packet, without CSMA/CA (i.e. send download data packet immediately after ACK), to the device. The IEEE 802.15.4 standard 1, 2] allows transmission of download data packet without CSMA/CA. The communication sequences for these download methods are described in Fig. 1(b) After receiving the download data packet, the device sends an acknowledgment packet which notifies the successful reception of the download data packet.

\section{Analysis for a Device with Download Traffic Only}

Let $n$ sensor devices be associated with the PAN coordinator. For download in IEEE 802.15.4 non-beacon mode, a device sends a download request packet to the PAN coordinator periodically to check whether there is a download data packet at the PAN coordinator. We assume that a device generates a download request packet after fixed number $I$ of slots from the moment of the completion of download procedure (See Fig. 1(b) . We also assume that the PAN coordinator generates a download data packet destined to the tagged device according to Poisson process with rate $\lambda_{\mathrm{d}}$ and the PAN coordinator can accommodate only one packet for the tagged device. So, if the tagged device has a packet to transmit then arriving packet is discarded. This assumption is reasonable in the case of infrequent packet's arrival in practical applications. The MAC sublayer will retry backoff process to send the packet until the backoff stage reaches to $M(=$ macMaxCSMABackoffs -1$)$. At the $M$ th backoff stage, if one of two CCAs fails, the packet is discarded. We assume that if the transmitted packet suffers collision, it will restart from the 0th backoff stage.

\subsection{Mathematical Model}

Let $s(t), 0 \leq s(t) \leq M$, be the backoff stage and $b(t)$ be the backoff counter. Let $(s(t), b(t))_{\mathrm{r}}$ denotes the backoff stage and backoff counter for download request packet. When the channel is idle at the first CCA, we define $b(t)=-1$. We assume that size of download request packet is fixed $R$ in the unit of slots. Let $T x_{\mathrm{r}}[k], 1 \leq k \leq R$, represent the state of the $k$ th slot of download request 
packet transmission. Let $R x_{\mathrm{d}}$ represent the state of download data packet in transmission. We assume that the length of data packet measured in slots is geometrically distributed with mean $\frac{1}{1-P_{\mathrm{Rx}, \mathrm{d}}}$. The switching time needs 1 slot and ACK packet needs 1slot. Let $(-1$, Switch) and $(-1, A C K)$ be the switching state and ACK state. Let $i d l e[k], 1 \leq k \leq I$, represent the state of the $k^{\text {th }}$ slot from the start of duration of fixed length $I$ for generating download request packet. Define $Y(t)$ at $t$ by :

$Y(t)=\left\{\begin{array}{l}i d l e[k], \text { when a device is in the state before generating download request packet } \\ (s(t), b(t))_{\mathrm{r}}, \text { when a device is in the process of backoff for download request packet } \\ (s(t),-1)_{\mathrm{r}}, \text { when channel is idle at the first CCA for download request packet } \\ T x_{\mathrm{r}}[k], \text { when a device transmits a download request packet } \\ (-1, S w i t h c h)_{\mathrm{r}}, \quad \text { when a device is waiting ACK for download request packet } \\ (-1, A C K)_{\mathrm{r}}, \text { when a device is receiving ACK for download request packet } \\ R x_{\mathrm{d}} \text { when a device receives a download data packet } \\ (-1, S w i t h c h)_{\mathrm{d}}, \quad \text { when PAN coordinator is waiting ACK for download data packet } \\ (-1, A C K)_{\mathrm{d}}, \text { when PAN coordinator is receiving ACK for download data packet }\end{array}\right.$

Then $Y(t)$ is a discrete Markov chain with one-step transition probabilities described in Fig. 2 for download procedure. Let $\pi_{i d l e[k]}, \pi_{(i, j)_{\mathrm{r}}}, \pi_{(i,-1)_{\mathrm{r}}}, \pi_{T x_{\mathrm{r}}[k]}$, $\pi_{(-1, \text { Switch })_{\mathrm{r}}}, \pi_{(-1, A C K)_{\mathrm{r}}}, \pi_{R x_{\mathrm{d}}}, \pi_{(-1, \text { Switch })_{\mathrm{d}}}$ and $\pi_{(-1, A C K)_{\mathrm{d}}}$ be the steady-state probability.

Next we will calculate the probability $\alpha$ of channel being busy at the first CCA, the probability $\beta$ of channel being busy at the second CCA and the probability $P_{s}$ of successful packet transmission.

Since the probability of the channel being idle at the first CCA for the given device is equal to the probability that the all other $n-1$ devices are

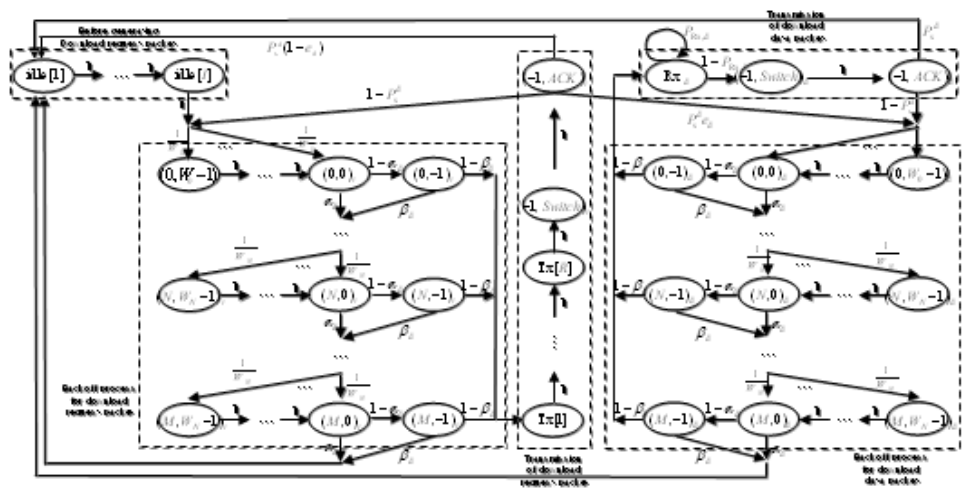

Fig. 2. Markov Chain for Download 
not in the states of $T x_{\mathrm{r}}[k],(-1, \text { Switch })_{\mathrm{r}},(-1, A C K)_{\mathrm{r}}, R x_{\mathrm{d}},(-1, \text { Switch })_{\mathrm{d}}$ and $(-1, A C K)_{\mathrm{d}}$. Therefore $\alpha$ is given by :

$$
\alpha=1-\left(1-\pi_{\mathrm{d}}\right)^{n-1},
$$

where

$$
\pi_{\mathrm{d}}=\sum_{k=1}^{R} \pi_{T x_{\mathrm{r}}[k]}+\pi_{R x_{\mathrm{d}}}+\sum_{j=0}^{A}\left(\pi_{(-1, S w i t c h)_{\mathrm{r}}}+\pi_{(-1, A C K)_{\mathrm{r}}}+\pi_{(-1, k)_{\mathrm{d}}}+\pi_{(-1, A C K)_{\mathrm{d}}}\right) .
$$

Note that in order to be eligible to sense the channel at the second CCA, the channel must be idle at the first CCA. So $\beta$ is the probability that the channel is busy when the tagged device senses at the second CCA, given that the channel is idle at the first CCA, i.e,

$1-\beta=P\{$ channel is idle at the second CCA $\mid$ channel is idle at the first CCA $\}$

$$
\begin{aligned}
& =\frac{P\{\text { channel is idle at the first CCA, channel is idle at the second CCA }\}}{P\{\text { channel is idle at the first CCA }\}} \\
& =\frac{\left(1-\pi_{\mathrm{d}}-\sum_{i=0}^{M} \pi_{(i,-1)_{\mathrm{r}}}\right)^{n-1}}{1-\alpha}
\end{aligned}
$$

The successful transmission probability, $P_{s}$, can be represented by :

$P_{s}=P\{$ successful transmission $\mid$ channel is idle at both the first CCA and the second CCA $\}$

$$
=\frac{\left\{1-\pi_{\mathrm{d}}-\sum_{i=0}^{M}\left(\pi_{(i, 0)_{\mathrm{r}}}+\pi_{(i,-1)_{\mathrm{r}}}\right)\right\}^{n-1}}{\left(1-\pi_{\mathrm{d}}-\sum_{i=0}^{M} \pi_{(i,-1)_{\mathrm{r}}}\right)^{n-1}}
$$

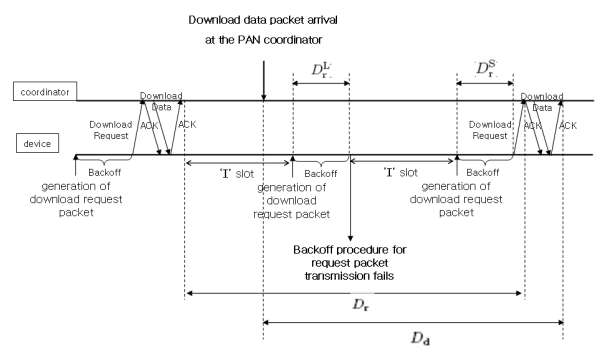

Fig. 3. Description of $D_{r}^{\mathrm{L}}, D_{r}^{\mathrm{S}} D_{r}$ and $D_{d}$

Let $e_{\mathrm{d}}$ be the probability that there is a download data packet at the PAN coordinator when download request packet arrives at the PAN coordinator. This event occurs when download data packet arrives during the time duration, $D_{\mathrm{r}}$, from the completion of one download procedure to the next arrival of download 
request packet at the PAN coordinator (See Fig. 3). The expected delay $\mathrm{E}\left[D_{\mathrm{r}}\right]$ are calculated by :

$$
\mathrm{E}\left[D_{\mathrm{r}}\right]=\sum_{k=0}^{\infty}\left(P_{\text {loss }}\right)^{k}\left(1-P_{\text {loss }}\right) \cdot\left\{k\left(I \cdot \sigma+\mathrm{E}\left[D_{\mathrm{r}}^{\mathrm{L}}\right]\right)+\left(I \cdot \sigma+\mathrm{E}\left[D_{\mathrm{r}}^{\mathrm{S}}\right]\right)\right\}+R \cdot \sigma
$$

where $P_{\text {loss }}$ is the probability of losing download request packet (given by (9)) and $\sigma$ is the length of a slot. The expected delay $\mathrm{E}\left[D_{\mathrm{r}}^{\mathrm{L}}\right]$ from the moment of generation of download request packet to the moment of discarding the packet and the expected delay $\mathrm{E}\left[D_{\mathrm{r}}^{\mathrm{S}}\right]$ from the moment of generation of download request packet to the moment of beginning of download request packet transmission are calculated in Appendix. So, $e_{\mathrm{d}}$ is approximately calculated using $\mathrm{E}\left[D_{\mathrm{r}}\right]$.

$$
e_{\mathrm{d}} \approx 1-e^{-\lambda_{\mathrm{d}} \cdot \mathrm{E}\left[D_{\mathrm{r}}\right]}
$$

To check the accuracy of the approximation (6), we simulated the system and it turns out that the approximation (6) is quite good (See Fig. 4).

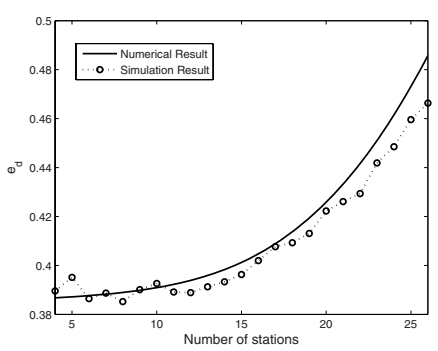

Fig. 4. Numerical and simulation results for $e_{d}$

Note that $\alpha, \beta, P_{s}$ and $e_{d}$ in (2), (3), (44) and (6) express in terms of steadystate probability and vice versa. Therefore by solving nonlinear equation of (2), (3), (4), (6), balance equations of this Markov Chain and normalization condition, we obtain all necessary values such as steady-stae probability, $\alpha$ and $\beta$.

\subsection{Performance Measures}

In this subsection, we obtain several performance measures such as throughput, delay, loss probability and energy consumption.

Throughput. The normalized system throughput $S$, defined as the fraction of time the channel is used to transmit download data packet successfully, is given as follows.

$$
S=n \cdot \pi_{R x_{\mathrm{d}}} \cdot P_{s}
$$


Delay. The expected delay $\mathrm{E}\left[D_{\mathrm{d}}\right]$ from the moment of download data packet arrival at the PAN coordinator to service completion point is approximately calculated by :

$$
\mathrm{E}\left[D_{\mathrm{d}}\right] \approx \mathrm{E}\left[D_{\mathrm{r}}\right]+\frac{1}{1-P_{\mathrm{Rx}, \mathrm{d}}}+2 A-\frac{\int_{0}^{\mathrm{E}\left[D_{\mathrm{r}}\right]} x \cdot \lambda_{\mathrm{d}} e^{-\lambda_{d} x} d x}{1-e^{-\lambda_{\mathrm{d}} \mathrm{E}\left[D_{\mathrm{r}}\right]}}
$$

The last term in the right-hand side represents the average duration from the completion of previous download procedure to a arrival of next download data packet.

Packet Loss Probability. Let the probabilities of losing download request packet denoted by $P_{\text {loss }}^{\mathrm{r}}$. Then we have

$$
\begin{aligned}
P_{\mathrm{loss}}^{\mathrm{r}}= & \sum_{v=0}^{M} \sum_{w=0}^{v}{ }_{v} \mathrm{C}_{w} \alpha^{w}\{(1-\alpha) \beta\}^{v-w}(1-\alpha)(1-\beta)\left(1-P_{s}\right) P_{\mathrm{loss}}^{\mathrm{r}} \\
& +\sum_{w=0}^{M}{ }_{M} \mathrm{C}_{w} \alpha^{w}\{(1-\alpha) \beta\}^{M-w}\{\alpha+(1-\alpha) \beta\}
\end{aligned}
$$

The general term in the first summation of (9) is the probability that the packet suffers loss after collision at the $v^{\text {th }}$ backoff stage in the first backoff procedure. Note that after collision the procedure starts from the $0^{\text {th }}$ backoff stage again. The second term of (9) is the probability that the packet in the first backoff procedure suffers loss because channel is busy at the first CCA or the second CCA at the $M^{\text {th }}$ backoff stage.

After a download data packet arrived in PAN coordinator waits until the download request packet is successfully transmitted from the tagged device and the download data packet is always successfully transmitted because the PAN coordinator transmits the download data packet without CSMA/CA.

Energy Consumption. Since power is quite critical in a sensor network, energy consumption is the most important performance measure. To obtain the total lifetime of a battery, we need a concept of average energy consumption $E^{\text {slot }}$ per one slot(mJ/slot). Let $E_{\text {idle }}, E_{T x}$ and $E_{R x}$ be the energy consumption for idle slot, transmission slot and reception(or CCA) slot, respectively. Since energy consumption for reception slot and CCA slot are equal, we do not distinguish the valus. Let $a^{\text {idle }}, a^{T x}$ and $a^{R x}$ be the probabilities of slot being idle, being transmission, being reception(or CCA). Then,

$$
\begin{aligned}
& a^{\text {idle }}=\sum_{k=1}^{I} \pi_{i d l e[k]}+\sum_{i=0}^{M} \sum_{j=1}^{W_{i}-1} \pi_{(i, j)_{\mathrm{r}}} \\
& a^{T x}=\pi_{T x_{\mathrm{r}}[k]}+\sum_{j=0}^{A} \pi_{(-1, k)_{\mathrm{d}}} \\
& a^{R x}=1-a^{\text {idle }}-a^{T x}
\end{aligned}
$$


Note that a device consumes $E_{R x}$ per one slot when it waits the download data from PAN coordinator.

The average energy consumption $E^{\text {slot }}$ per one slot is obtained as follows.

$$
E^{\text {slot }}=a^{\text {idle }} E_{\text {idle }}+a^{T x} E_{T x}+a^{R x} E_{R x}
$$

\section{Numerical and Simulation Results for Both Upload and Download Traffic}

In this section, numerical results and simulation results for performance measures of the network in the same environments as in 4 are presented to compare the results of backoff method with one of piggyback method. For our numerical results, $I$ is set to 500 backoff slots. The average length of a download data packet, $\frac{1}{1-P_{\mathrm{Rx}, \mathrm{d}}}$, is set to 4 . Note that $\sigma=0.32 \mathrm{~ms}$ in case of $250 \mathrm{Mbps}, 2.4 \mathrm{GHz}$. $N$ and $M$ are 2 and 4 , respectively. $W_{0}$ is set to $2^{3}=8$ in our experiment. The energy consumptions at $T_{x}, R_{x}$, and CCA states are $0.0100224 \mathrm{~mJ}, 0.0113472 \mathrm{~mJ}$ and $0.0113472 \mathrm{~mJ}$, respectively, [6]. A device consumes $0.000056736 \mathrm{~mJ}$ during idle state.

Fig. 5(a) depicts the expected delay $\mathrm{E}\left[D_{\mathrm{d}}\right]$ for download traffic of backoff method and piggyback method. As the number of devices increases, $\mathrm{E}\left[D_{\mathrm{d}}\right]$ for two methods increase due to the exponential backoff by competitions of each other. Fig. 5(b) depicts the packet loss probability for download traffic of backoff method and piggyback method, respectively. Also $P_{\text {loss }}^{d}$ for backoff method increases as the number of devices increases. As mentioned in 3.2 , the download packet loss does not occur in piggyback method. Fig. 5(c) depicts the average energy consumption $E^{\text {slot }}$ per one backoff slot of both backoff method and piggyback method. Fig. [5 shows that the numerical results and simulation results for performance measures differ slightly. This may be caused by the analytical model where two approximations (6) and (8) are used. Numerical results and simulation results show that by removing a backoff procedure in the backoff method, the piggyback method can slightly reduce the delay for download, loss

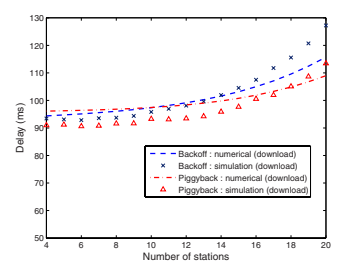

(a) Expected delay for upload and download
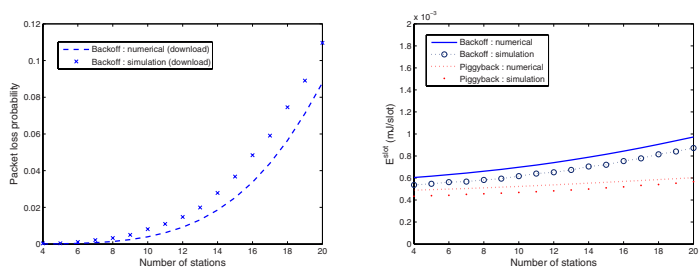

(b) Packet loss probabil- (c) Energy consumption ity for upload and down- for a device load

Fig. 5. Numerical and simulation Results : Performance measures 
probability download packet is prevented. Especially, the reduction of energy consumption is remarkable.

Finally, our results are used for determining the optimal number of devices which can be accommodated in the system while supporting the required QoS on the expected packet delay and the packet loss probability. For instance, with the requirements of $\mathrm{E}\left[D_{\mathrm{d}}\right] \leq 110 \mathrm{~ms}$ and $P_{\text {loss }}^{d} \leq 2 \%$, the optimal number of devices in the network is obtained as 20 in backoff method and 13 in piggyback method from Fig. 5(a) and Fig. 5(b), From these results, we can perceive that the new scheme which reduce the delay for download data packet is necessary. With this case, we obtain from Fig. 5(c) that the average energy consumption $E^{\text {slot }}$ per one backoff slot is $7.1 \times 10^{-4} \mathrm{~mJ} /$ slot in backoff method and $5.3 \times 10^{-4} \mathrm{~mJ} / \mathrm{slot}$ in piggyback method.

Acknowledgments. This research is supported by the MIC, under the ITRC support program supervised by the IITA.

\section{References}

1. IEEE 802.15.4, Wireless LAN Medium Access Control(MAC) and Physical Layer (PHY) specifications for Low-Rate Wireless Personal Area Network (LR-WPANs) (2003)

2. IEEE 802.15.4, Wireless LAN Medium Access Control(MAC) and Physical Layer (PHY) specifications for Low-Rate Wireless Personal Area Network (LR-WPANs) (2006)

3. Kim, T.O., Kim, H., Lee, J., Park, J., Choi, B.D.: Performance Analysis of IEEE 802.15.4 with Non-Beacon-enabled CSMA/CA in Non-Saturated Condition. In: Sha, E., Han, S.-K., Xu, C.-Z., Kim, M.H., Yang, L.T., Xiao, B. (eds.) EUC 2006. LNCS, vol. 4096, Springer, Heidelberg (2006)

4. Kim, T.O., Park, J., Choi, B.D.: Analytic Model of IEEE 802.15.4 with Download Traffic. In: Proceeding of The Second IEEE International Symposium on Pervasive Computing and Ad Hoc Communications (PCAC-07) (May 2007)

5. Pollin, S., Ergen, M., Ergen, S.C., Bougard, B., der Perre, L.V., Catthoor, F., Moerman, I., Bahai, A., Varaiya, P.: Performance Analysis of the Slotted IEEE 802.15.4 Medium Access Layer, draft-jwl-tcp-fast-01.txt (2005)

6. Park, T., Kim, T., Choi, J.Y., Choi, S., Kwon, W.: Throughput and Energy Consumption Analysis of IEEE 802.15.4 Slotted CSMA/CA. Electronics Letters (2005)

7. Misic, J., Shafi, S., Misic, V.B.: Performance of a Beacon Enabled IEEE 802.15.4 Cluster with Downlink and Uplink Traffic. IEEE Transactions on Paraller and Distributed systems 17(4) (April 2006)

\section{Appendix: Delay for Download}

In this section, we obtain the expected durations $\mathrm{E}\left[D_{\mathrm{r}}^{\mathrm{L}}\right], \mathrm{E}\left[D_{\mathrm{r}}^{\mathrm{S}}\right]$ and $\mathrm{E}\left[D_{\mathrm{d}} *\right]$ (See Fig. 3). $\mathrm{E}\left[D_{\mathrm{r}}^{\mathrm{L}}\right]$ is the expected time duration from the moment of generation of download request packet to the moment of discarding the packet, and $\mathrm{E}\left[D_{\mathrm{r}}^{\mathrm{S}}\right]$ is the expected time duration from the moment of generation of download request 
packet to the moment of beginning of download request packet transmission. To obtain $\mathrm{E}\left[D_{\mathrm{r}}^{\mathrm{L}}\right]$ and $\mathrm{E}\left[D_{\mathrm{r}}^{\mathrm{S}}\right]$, let $P^{\mathrm{c}}$ be the probability that a packet suffers collision in a backoff procedure. Then,

$$
P^{\mathrm{c}}=\sum_{v=0}^{M} \sum_{r=0}^{v}{ }_{v} \mathrm{C}_{r} \alpha^{r}\{(1-\alpha) \beta\}^{v-r}(1-\alpha)(1-\beta)\left(1-P_{\mathrm{S}}\right) .
$$

Let $\mathrm{E}\left[D_{\text {backoff }}^{\mathrm{T}}\right]$ and $\mathrm{E}\left[D_{\text {backoff }}^{\mathrm{L}}\right]$ be the expected number of backoff slots that a packet experience until the moment of transmission attempt in a backoff procedure and the expected number of backoff slots that a packet experience until the moment of discarding in a backoff procedure, respectively. Then,

$$
\mathrm{E}\left[D_{\text {backoff }}^{\mathrm{T}}\right]=\frac{\sum_{v=0}^{M} \sum_{r=0}^{v}{ }_{v} \mathrm{C}_{r} \alpha^{r}\{(1-\alpha) \beta\}^{v-r}\left(\sum_{i=0}^{v} \frac{W_{i}-1}{2}+2 v-r+2\right)}{\sum_{v=0}^{M} \sum_{r=0}^{v}{ }_{v} \mathrm{C}_{r} \alpha^{r}\{(1-\alpha) \beta\}^{v-r}}
$$

$$
\begin{aligned}
\mathrm{E}\left[D_{\text {backoff }}^{\mathrm{L}}\right]= & \frac{\sum_{r=0}^{M}{ }_{M} \mathrm{C}_{r} \alpha^{r}\{(1-\alpha) \beta\}^{M-r}}{\sum_{r=0}^{M+1}{ }_{M+1} \mathrm{C}_{r} \alpha^{r}\{(1-\alpha) \beta\}^{M+1-r}} \\
& \quad \times\left\{\alpha\left(\sum_{i=0}^{M} \frac{W_{i}-1}{2}+2 M-r+1+(1-\alpha) \beta\left(\sum_{i=0}^{M} \frac{W_{i}-1}{2}+2 M-r+2\right)\right\}\right.
\end{aligned}
$$

Note that a download packet is discarded when the CCA fails at the $M$ th backoff stage. So, the expected duration $\mathrm{E}\left[D_{\mathrm{r}}^{\mathrm{L}}\right]$ is given by :

$$
\mathrm{E}\left[D_{\mathrm{r}}^{\mathrm{L}}\right]=\sum_{k=0}^{\infty}\left(P^{c}\right)^{k}\left(1-P^{c}\right)\left\{k\left(D_{\text {backoff }}^{\mathrm{T}}+R+A\right)+D_{\text {backoff }}^{\mathrm{L}}\right\} \sigma .
$$

The general term in (14) is the expected duration for the case that a packet is discarded after the $k$ th collision. Similarly, the expected duration $\mathrm{E}\left[D_{\mathrm{r}}^{\mathrm{S}}\right]$ is given by :

$$
\mathrm{E}\left[D_{\mathrm{r}}^{\mathrm{S}}\right]=\sum_{k=0}^{\infty}\left(P^{c}\right)^{k}\left(1-P^{c}\right)\left\{k\left(D_{\text {backoff }}^{\mathrm{T}}+R+A\right)+D_{\text {backoff }}^{\mathrm{T}}\right\} \sigma .
$$

The general term in (15) is the expected duration for the case that a packet is successfully transmitted after the $k$ th collision. 